\title{
Pillars to improve patient outcomes: training and assessment methods for surgery
}

\author{
Andrew J. Hung ${ }^{1} \cdot$ Ahmed Ghazi $^{2}$ \\ Published online: 3 June 2020 \\ ○) Springer-Verlag GmbH Germany, part of Springer Nature 2020
}

Surgeon performance directly impacts patient outcomes [1]. Training and assessment go hand in hand-one cannot exist without the other. Training by nature requires formative feedback, otherwise it is reduced to sheer repetition. Naturally, the method and quality of assessment influences a learning surgeon's progress during training. We have commissioned a balanced library of eight articles - half with an emphasis on surgical assessment, while the other half have focused on training. There is a heavy emphasis on robotic surgery, but the concepts are largely translatable across surgical approaches.

Four papers in this issue focus on surgical assessment. In their original research, Nguyen et al. showed that automated performance metrics (APMs) and task-evoked pupillary response (a measure of cognitive workload) can distinguish surgeon expertise levels during robotic surgical tasks [2]. Furthermore, under high cognitive workload, there can be a divergence in robotic movement profiles between expertise levels. Under increased cognitive workload, experienced surgeons tend to slow their instrument and camera movement. In contrast, learning surgeons tend to speed up under increased cognitive workload. This paper is the first to demonstrate differences in surgeon behavior based on cognitive workload. More work in this space may help us better provide timely feedback to learning surgeons.

$\mathrm{Wu}$ et al. evaluated the technical variability in the approach to a robot-assisted radical prostatectomy across

Andrew J. Hung

Andrew.Hung@med.usc.edu

Ahmed Ghazi

Ahmed_Ghazi@urmc.rochester.edu

1 Center for Robotic Simulation and Education, Catherine and Joseph Aresty Department of Urology, University of Southern California Institute of Urology, 1441 Eastlake Avenue, Suite 7416, Los Angeles, CA 90033, USA

2 Simulation Innovation Laboratory, Department of Urology, University of Rochester Medical Center, 601 Elmwood Ave, Rochester, NY 14642, USA a statewide surgical collaborative [3]. The authors found that despite worldwide popularity of RARP, the operation across a large geographical region of the US is still far from standardized. Nonetheless, while peri-operative outcomes were not correlated with surgeon's operative time, surgeons with greater estimated blood loss deviated most from the norm of the techniques employed. Perhaps some degree of standardization could improve patient outcomes.

Chen et al. compared performance measures of RARP cases involving trainees and those without [4]. Automated performance metrics, derived from systems data of the robot, indicate less overall efficiency of the RARP when trainees perform at least one of the designated "cardinal steps." That said, the degree of trainee involvement does not significantly affect major clinical outcomes. Presumably, this relies on vigilant supervision by faculty surgeons, guaranteeing that surgery is performed to their standards.

Finally, Goldberg highlighted several studies from multiple surgical specialties and procedures that have confirmed the association between surgeon skill, measured in varying methods, and clinically significant patient outcomes [1]. Establishing this relationship between surgeon performance and patient outcomes asserts the value and importance of surgeon assessment in clinical and training environments.

The above articles focused on assessment methods and highlighted novel objective measures of surgeon performance (APMs measuring efficiency), biometric measures of surgeon cognitive workload, surgeon variability in technique, and finally, how some of these measures may relate to patient outcomes. In the near future, we should expect to see greater focus on objectively measuring true technical skill and not mere efficiencies. Most measures of technical skill today require manual observation and evaluation by content experts. Presently, these measures cannot be done automatically and completely objectively. At least, presently, perhaps a balance of objective measures of efficiency and manual assessment of skills is necessary to provide a full picture of surgeon performance. 
The following papers on training methods underline the importance of how surgeons are trained, especially in consideration of training curriculums and environments. In the era where residents are required to learn an increasing number of procedures to competency, without the same total number of hours available for training, Gurung et al. focused on minimizing valuable simulation-training time utilizing a more effective strategy for proficiency-based virtual reality (VR) robotic training [5]. Their Accelerated Skills Acquisition Protocol (ASAP) focused on proficiency training of graded VR exercises at only the uppermost difficulty level. The ASAP group required only half the repetitions to reach proficiency compared to conventional training but with no significant differences in transfer and retention tasks at 3, 6 and 12 months. The authors concluded that ASAP proved to be a time-efficient and effective training approach, without compromising the acquisition or retention of technical surgical skills which may have significant implications on the design of simulation curricula.

Other struggles in surgical simulation includes the identification of the ideal training platform for advanced procedural training that combines the relevant anatomy found in cadavers and live tissue properties including bleeding found in living animal models. Melynk et al. demonstrated a novel, perfused high-fidelity, anatomical, polymer-based kidney model for robot-assisted partial nephrectomy (RAPN) simulation, fabricated using a combination of $3 \mathrm{D}$ printing and hydrogel casting [6]. They present a series of extensive bio-mechanical tests that identified the ideal hydrogel formula which replicates tissue characteristics (elasticity, strain and pliability) of various living kidney tissue components as well as functional properties in the partial nephrectomy simulation (replicating perfusion during tumor resection and suturing forces during renorrhaphy). This is the first study to utilize extensive material testing analyses to determine the mechanical and functional properties of a perfused, inanimate simulation platform for RAPN which could soon become a standard for advanced robotic simulation-based training.

Justin C \& Wisz P in 'Training in robotic surgery, replicating the airline industry. How far have we come?' reviewed current robotic training curricula with training in aviation, in an attempt to evaluate similarities and to provide insights into how healthcare can further learn from successful initiatives in aviation training [7]. While many similarities exist, international standards for training curricula, simulation devices and instructors present in aviation are lacking in surgery. Pilots and career instructors are mandated to complete re-qualification with benchmarked high stakes tests, which are also notoriously lacking in surgical standards. The authors conclude that although many aviation training initiatives have been replicated in surgical training, there remain considerable differences in regulations and standards. The adoption of these established and proven aviation methods of assessment and regulation could help robotic surgical training become more efficient, effective and ultimately safer.

Similarly, Griffin et al. reviewed the standardization of non-technical skills (NTS) curriculum for evaluation and training in Urology [8]. The authors recommend simulation training as the most effective way to train NTS through the employment of several best practices including training in multiple settings (e.g. wards, clinics and operating rooms) with an inter-professional team and debriefing after training. The authors also alluded to the new challenges robotic surgery brings to NTS training including the physical displacement of the surgeon from their team, a change in the surgeons' role, and the addition of members requiring the team to adapt their interactions and maintain excellent situational awareness and communication, including consistent awareness of patient status and equipment failure.

As surgical approaches evolve, so do the methods of training. All involved parties have the duty of providing the greatest possible care for their patients using standardized, consensus-based training paradigms developed from other high-stakes industries with a proven track record in safety standards.

In total, these papers on assessment and training represent some of the cutting-edge research in either domain, and the narrative reviews help shed light on the big picture. How we assess and train our surgeon's matters, as they are truly the pillars to improve patient outcomes.

\section{References}

1. Goldenberg MG (2019) Evidence that surgical performance predicts clinical outcomes. World J Urol. https://doi.org/10.1007/ s00345-019-02857-w

2. Nguyen JH, Chen J, Marshall SP, Ghodoussipour S, Chen A, Gill IS, Hung AJ (2019) Using objective robotic automated performance metrics and task-evoked pupillary response to distinguish surgeon expertise. World J Urol. https://doi.org/10.1007/s0034 5-019-02881-w

3. Wu RC, Prebay ZJ, Patel P, Kim T, Qi J, Telang J, Linsell S, Kleer E, Miller DC, Peabody JO, Ghani KR, Johnston WK 3rd, Michigan Urological Surgery Improvement C (2019) Using video review to understand the technical variation of robot-assisted radical prostatectomy in a statewide surgical collaborative. World J Urol. https://doi.org/10.1007/s00345-019-02906-4

4. Chen A, Ghodoussipour S, Titus MB, Nguyen JH, Chen J, Ma $\mathrm{R}$, Hung AJ (2019) Comparison of clinical outcomes and automated performance metrics in robot-assisted radical prostatectomy with and without trainee involvement. World J Urol. https://doi. org/10.1007/s00345-019-03010-3

5. Gurung PMS, Campbell T, Wang B, Joseph JV, Ghazi AE (2019) Accelerated Skills Acquisition Protocol (ASAP) in optimizing robotic surgical simulation training: a prospective randomized study. World J Urol. https://doi.org/10.1007/s00345-019-02858-9

6. Melnyk R, Ezzat B, Belfast E, Saba P, Farooq S, Campbell T, McAleavey S, Buckley M, Ghazi A (2019) Mechanical and 
functional validation of a perfused, robot-assisted partial nephrectomy simulation platform using a combination of 3D printing and hydrogel casting. World J Urol. https://doi.org/10.1007/s0034 5-019-02989-z

7. Collins JW, Wisz P (2019) Training in robotic surgery, replicating the airline industry. How far have we come? World J Urol. https:// doi.org/10.1007/s00345-019-02976-4

8. Griffin C, Aydin A, Brunckhorst O, Raison N, Khan MS, Dasgupta P, Ahmed K (2019) Non-technical skills: a review of training and evaluation in urology. World J Urol. https://doi.org/10.1007/s0034 5-019-02920-6

Publisher's Note Springer Nature remains neutral with regard to jurisdictional claims in published maps and institutional affiliations. 